\title{
On the Origins of Sub-Subgiants: Mass Transfer, Dynamical Encounters, and Magnetic Fields
}

\author{
Emily M. Leiner* $\dagger$ \\ University of Wisconsin-Madison \\ E-mail: leinerdastro.wisc.edu
}

\section{Aaron M. Geller}

Northwestern University-CIERA \& the Adler Planetarium

E-mail: a-geller@northwestern.edu

\section{Robert Mathieu}

University of Wisconsin-Madison

E-mail: mathieudastro.wisc.edu

In an optical color-magnitude diagram sub-subgiant stars (SSGs) lie redward of the mainsequence and fainter than the red giant branch in a region not easily populated by standard stellar evolution pathways. The first SSGs were discovered in the open cluster M67 [1; 2]. Subsequent discoveries of dozens of SSGs in both open and globular clusters solidifies these stars as their own unique class sharing several properties: X-ray emission, photometric variability, and, where binary status is known, orbital periods between 2-18 days. While there has been speculation on what mechanisms may create these unusual stars, no well-developed theory has yet been proposed to explain their origins. Here we focus on three possible theories of SSG formation: (1) mass transfer in a binary system, (2) mass loss in a dynamical encounter, (3) a reduced luminosity due to magnetic activity that lowers convective efficiency. We compare the expected stellar and orbital properties of these models with the six known SSGs in the two open clusters M67 and NGC 6791.

Frank N. Bash Symposium 2015

18-20 October

The University of Texas at Austin, USA

\section{*Speaker.}

${ }^{\dagger}$ This research was supported by a Grant-In-Aid of Research from Sigma Xi, the Scientific Research Society and by NASA through grants HST- AR-13910 and HST-GO-13354.001-A from the Space Telescope Science Institute, which is operated by AURA, Inc., under NASA contract NAS 5-26555. 


\section{Introduction}

We use the stellar evolution code Modules for Experiments in Stellar Astrophysics (MESA) [3] to create models of SSGs formed via three possible formation mechanisms. We compare the properties of our models to a sample of 6 SSGs in open clusters M67 and NGC 6791 (see Figure 1) for which $3 \mathrm{D}$ kinematic memberships and orbital solutions have been determined.
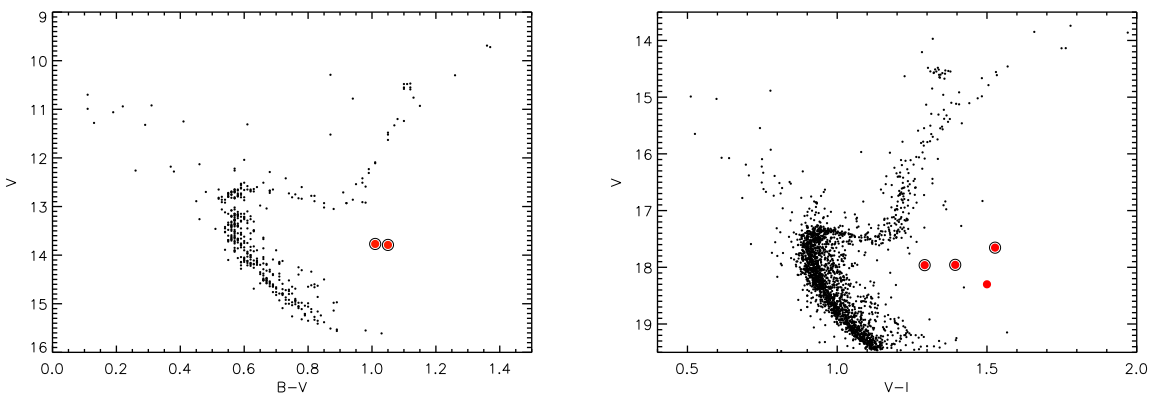

Figure 1: (left) BV CMD of the open cluster M67 showing all 3D kinematic members [4]. (right) VI CMD of the open cluster NGC 6791 showing all proper-motion members [5]. 3D kinematic member SSGs $[4,6]$ are shown with red circles. Binary SSGs are also circled in black.

\section{SSG Formation Mechanisms}

Mass Transfer: Mass transfer in a binary may create SSGs. If the primary overflows its Roche lobe as it evolves off the main sequence, it loses mass creating smaller and less luminous subgiants (see Figure 2, red line). However, our models show these mass transferring stars can only have periods up to 3.36 days in NGC 6791 and 5.49 days in M67, too short to explain all but one of the SSGs in our sample. We can produce the period of the shortest period SSG (2.8 days), but not with its observed mass ratio $(q=0.7)$. We conclude that while mass transfer can produce stars in the SSG region, it does so infrequently and cannot produce the systems observed in M67 and NGC 6791.

Subgiant dynamical encounters: Another scenario to create an SSG invokes an off-center dynamical encounter in which a subgiant loses substantial envelope material but does not collide with a passing star (see Figure 2, blue line). While this could produce stars in the SSG CMD region, such encounters are rare in open cluster environments. In globular clusters this may be an important mechanism of SSG formation.

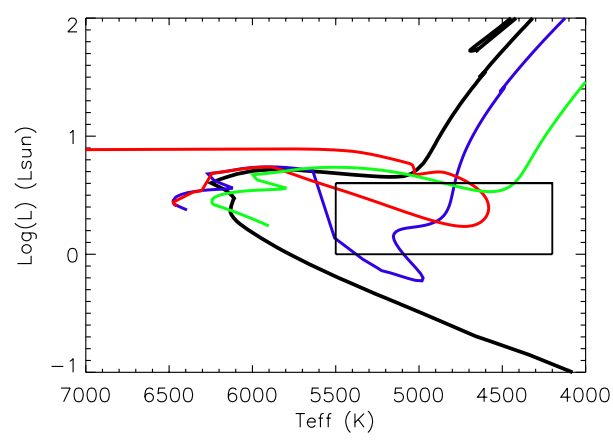

Figure 2: MESA evolutionary tracks for SSGs created via mass transfer in a 1.0 day period binary (red), a dynamical encounter in which a $1.3 \mathrm{M}_{\odot}$ subgiant rapidly loses $0.5 \mathrm{M}_{\odot}$ from its envelope and then continues to evolve normally (blue), and a model with lowered convective mixing length to mimics the effect of magnetic fields (green). The solid black line shows a 4 Gyr Padova isochrone (the age of M67). The black rectangle indicates the region in which SSGs are found. 
Magnetic Fields: Five of the six SSGs in M67 and NGC 6791 are short period (2.8 -18.4 day) RS CVn-type binaries. These close binaries are rapidly rotating due to tidal synchronization and display signs of magnetic activity including X-ray emission, $\mathrm{H}$-alpha emission, and photometric variability $[7,8]$. Strong magnetic fields in these stars may inhibit convection, causing the stars to be slightly expanded, cooler, and covered in large starspots. MESA models indicate that lowering the mixing length coefficient (a proxy for less-efficient convection, [9]) from $\alpha=2.0$ to 1.2 can create larger, cooler giants or subgiants that evolve through the SSG region (see Figure 2, green line). If magnetic fields can lower the luminosity of subgiants in close binaries, this mechanism has by far the highest frequency of SSG production in open cluster environments.

We conclude that all three proposed formation mechanisms (mass transfer, dynamical encounters with subgiants, and magnetic fields) are capable of producing stars in the SSG region of a CMD. However, mass transfer and dynamical encounters do so with low frequency in open clusters and mass transfer can only produce shorter period binaries than those observed in M67 and NGC 6791. In globular clusters, these may be more important mechanisms. At open cluster masses, magnetic fields that reduce convective efficiency are the most likely candidate to explain the SSGs, though better physical models are needed. Other production mechanism not investigated here may also be possible, e.g. mergers of main-sequence stars due to dynamical encounters.

\section{References}

[1] T. Belloni, F. Verbunt, and R. D. Mathieu, X-rays from old open clusters: M 67 and NGC 188,339 (Nov., 1998) 431-439, [astro-ph/9808329].

[2] R. D. Mathieu, M. van den Berg, G. Torres, et al., Sub-Subgiants in the Old Open Cluster M67?, 125 (Jan., 2003) 246-259, [astro-ph/0209568].

[3] B. Paxton, P. Marchant, J. Schwab, E. B. Bauer, L. Bildsten, M. Cantiello, L. Dessart, R. Farmer, H. Hu, N. Langer, R. H. D. Townsend, D. M. Townsley, and F. X. Timmes, Modules for Experiments in Stellar Astrophysics (MESA): Binaries, Pulsations, and Explosions, 220 (Sept., 2015) 15, [arXiv:1506.03146].

[4] A. M. Geller, D. W. Latham, and R. D. Mathieu, Stellar Radial Velocities in the Old Open Cluster M67 (NGC 2682). I. Memberships, Binaries, and Kinematics, 150 (Sept., 2015) 97, [arXiv:1507.01949].

[5] I. Platais, K. M. Cudworth, V. Kozhurina-Platais, et al., A New Look at the Old Star Cluster NGC 6791, 733 (May, 2011) L1, [arXiv: 1104 .5473].

[6] K. E. Milliman, E. L. Leiner, R. D. Mathieu, B. M. Tofflemire, and I. Platais, Spectroscopic Binary Orbits of Sub-Subgiants in NGC 6791, in prep, (2016).

[7] M. van den Berg, G. Tagliaferri, T. Belloni, and F. Verbunt, A Chandra observation of the old open cluster M 67, 418 (May, 2004) 509-523, [astro-ph/0311138].

[8] M. van den Berg, F. Verbunt, G. Tagliaferri, T. Belloni, L. R. Bedin, and I. Platais, A Chandra X-Ray Study of the Interacting Binaries in the Old Open Cluster NGC 6791, 770 (June, 2013) 98, [arXiv:1301.2331].

[9] G. Chabrier, J. Gallardo, and I. Baraffe, Evolution of low-mass star and brown dwarf eclipsing binaries, 472 (Sept., 2007) L17-L20, [arXiv: 0707 . 1792]. 